\title{
Expression profiling of choline and ethanolamine kinases in MCF7, HCT116 and HepG2 cells, and the transcriptional regulation by epigenetic modification
}

\author{
CHUA SIANG LING ${ }^{1}$, KHOO BOON YIN ${ }^{2}$, SEE TOO WEI CUN ${ }^{1}$ and FEW LING LING ${ }^{1}$ \\ ${ }^{1}$ School of Health Sciences, Universiti Sains Malaysia, Kubang Kerian, Kelantan 16150; \\ ${ }^{2}$ Institute for Research in Molecular Medicine, Universiti Sains Malaysia, \\ Penang 11800, Malaysia
}

Received December 10, 2013; Accepted September 4, 2014

DOI: $10.3892 / \mathrm{mmr} .2014 .2707$

\begin{abstract}
The function of choline kinase (CK) and ethanolamine kinase (EK) is to catalyse the phosphorylation of choline and ethanolamine, respectively, in order to yield phosphocholine (PCho) and phosphoethanolamine (PEtn). A high expression level of PCho, due to elevated CK activity, has previously been associated with malignant transformation. In the present study, a quantitative polymerase chain reaction was performed to determine the mRNA expression profiles of $c k$ and $e k$ mRNA variants in MCF7 breast, HCT116 colon and HepG2 liver cancer cells. The $c k$ and $e k$ mRNA expression profiles showed that total $c k \alpha$ was expressed most abundantly in the HepG2 cells. The HCT116 cells exhibited the highest $c k \beta$ and $e k 1$ mRNA expression levels, whereas the highest ek $2 \alpha$ mRNA expression levels were detected in the MCF7 cells. The $c k \beta$ variant had higher mRNA expression levels, as compared with total $c k \alpha$, in both the MCF7 and HCT116 cells. Relatively low ekl mRNA expression levels were detected, as compared with $e k 2 \alpha$ in the MCF7 cells; however, this was not observed in the HCT116 and HepG 2 cells. Notably, the mRNA expression levels of $c k \alpha 2$ were markedly low, as compared with $c k \alpha 1$, in all three cancer cell lines. The effects of epigenetic modification on $c k$ and $e k$ mRNA expression, by treatment of the cells with the histone deacetylase inhibitor trichostatin A (TSA), were also investigated. The results of the present study showed that the mRNA expression levels of $c k \alpha$, $c k \beta$ and $e k 2 \alpha$ were affected by TSA. An increase $>8$-fold was observed in $e k 2 \alpha$ mRNA expression upon treatment with TSA, in a concentration- and time-dependent manner. In conclusion, the levels of $c k$ and $e k$ transcript variants in the three cancer
\end{abstract}

Correspondence to: Dr Few Ling Ling, School of Health Sciences, Universiti Sains Malaysia, Kubang Kerian 16150, Kelantan, Malaysia E-mail: fewling@usm.my

Key words: choline kinase, ethanolamine kinase, mRNA expression, quantitative real-time PCR, histone deacetylase inhibitor, trichostatin A, epigenetic modification cell lines were varied. The effects of TSA treatment on the mRNA expression levels of $c k$ and $e k$ imply that $c k$ and $e k$ mRNA expression may be regulated by epigenetic modification.

\section{Introduction}

Cholinekinase (CK) and ethanolaminekinase(EK)are enzymes that initiate the first step in the Kennedy pathway, resulting in the biosynthesis of phosphatidylcholine and phosphatidylethanolamine (1). In the presence of $\mathrm{Mg}^{2+}, \mathrm{CK}$ and $\mathrm{EK}$ catalyse the ATP-dependent phosphorylation of choline and ethanolamine into phosphocholine (PCho) and phoshoethanolamine (PEtn), respectively. In humans, $\mathrm{CK}$ is encoded by two separate genes, $c k \alpha$ and $c k \beta$, which are located on chromosomes 11q13.2 and 23q13.33 (Ensembl Genome Browser v48, Gene view: http://www.ensembl.org/), respectively. The $c k \alpha$ transcript has been shown to undergo alternative splicing, resulting in the generation of two mRNA splice variants: $c k \alpha 1$ (NCBI reference: NM_212469.1) and ck 2 (NCBI reference: NM_001277.2). These splice variants are later translated into two functional protein isoforms, $\mathrm{CK} \alpha 1$ and $\mathrm{CK} \alpha 2$, consisting of 439 and 457 amino acids, respectively (2). The $c k \alpha 2$ variant contains an additional stretch of 54 nucleotides, as compared with the $c k \alpha l$ variant. $\mathrm{CK} \beta$ is translated from the $c k \beta$ (NCBI reference: NM_005198.4) mRNA transcript, and consists of 395 amino acids and the protein sequence has $60 \%$ homology with the $\mathrm{CK} \alpha 1$ and $\mathrm{CK} \alpha 2$ isoforms (2). Mammalian $\mathrm{CK} \beta$ was initially characterised in rat liver (3). In humans and mice, $\mathrm{CK} \beta$, alongside muscular carnitine-palmitoyl transferase $1 \mathrm{~b}$ (M-CPT1b), are encoded by a bicistronic gene (4). Due to the overlapping of the $c k \beta$ and $m$-cptlb genes on the chromosome; therefore, Yamazaki et al (5) previously suggested that these two genes may share the same regulators.

Human EK exists as three isoforms: EK1, EK $2 \alpha$ and EK $2 \beta$, encoded by two separate genes; $e k 1$, which produces the $e k 1$ transcript variant 1 (NCBI reference: NM_018638.4); and $e k 2$, which undergoes alternative splicing to produce $e k 2 \alpha$ (NCBI reference: NM_018208.2) and $e k 2 \beta$ (GenBank reference: AK001623.1) transcript variants. $e k 2 \alpha$ and $e k 2 \beta$ are respectively translated into EK $2 \alpha$ and EK2 $\beta$ (6). 
CK actively participates in cell proliferation, which is modulated by various kinases and metabolites derived from phospholipids. PCho is a crucial lipid metabolite associated with cell proliferation and tumor development. Previously, a significant increase in CK activity and elevation of PCho levels was shown to be associated with cancer progression (7). Malignant transformation and progression have previously been reported to alter phospholipid metabolism, by increasing the production of PCho $(8,9)$. A high concentration of PCho is a common characteristic of tumor-derived cells, and may be used as a novel biomarker for cancer diagnosis and assessment of cancer progression $(10,11)$. CK $\alpha$ has also been proposed as a potential prognostic marker, to predict the outcome in patients with non-small-cell lung cancer (12).

The aim of the present study was to determine and analyze the expression profiles of $c k$ and $e k$ mRNA variants in MCF7 breast, HCT116 colon and HepG2 liver cancer cells, using quantitative polymerase chain reaction (qPCR). The cell lines were chosen due to the previously reported differential expression of $\mathrm{CK} \alpha$ in breast, colon and liver tissues (13). Although $c k \alpha$ overexpression is usually associated with carcinogenesis $(12,14)$, it remains unclear whether this is always the case, as CK $\alpha$ protein expression levels have shown significant variation in numerous tumour tissues (13). The expression levels of $c k \beta$, relative to $c k \alpha$, have also been shown to affect the regulation of the cell cycle (15). Similarly, EK, which belongs to the same family of enzymes as CK, has been suggested to have a role in carcinogenesis, by promoting the growth of transformed cells (16). However, the expression levels of the $e k$ gene transcripts in various cancer cells have not yet been compared. Quantification of $c k$ and $e k$ expression levels in numerous cancer cells may provide a new insight into their roles in promoting cancer cell growth. In addition, the regulation of $c k$ and $e k$ transcriptional activities in cancer cells by epigenetic modification remains to be elucidated. Epigenetic modification, such as histone deacetylation, modulates the expression of numerous genes at the transcriptional level, including genes that encode tumor protein p53 (17,18), E2F (19) and nuclear factor- $\kappa \mathrm{B}(19,20)$. Numerous histone deacetylase inhibitors (HDACi) have been synthesised, including trichostatin A (TSA), which belongs to the hydroxamic acids group. HDACi have been shown to regulate gene transcription by inducing histone acetylation, and modulating the recruitment of transcription factors and other proteins to the promoter region of target genes (21). Notably, HDACi has also been reported to display significant demethylating activity by indirectly altering DNA and histone methylation (22). TSA has been demonstrated to indirectly induce gene promoter demethylation in fungi (23). To investigate whether the expression levels of $c k$ and $e k$ transcripts are affected by DNA methylation, a qPCR analysis was performed to determine the effects of TSA treatment in HepG2 cells.

\section{Materials and methods}

Cell culture. The MCF7 breast adenocarcinoma cell line [American Type Culture Collection (ATCC) no. HTB-22], HCT116 colorectal carcinoma cell line (ATCC no. CCL-247) and HepG2 liver hepatocellular carcinoma cell line
(ATCC no. HB-8065) were obtained from ATCC (Manassas, VA, USA). The cells were grown in Dulbecco's modified Eagle's medium (DMEM; Gibco Life Technologies, Carlsbad, CA, USA), supplemented with $10 \%$ fetal bovine serum (FBS, Gibco Life Technologies). The cell lines were maintained at $37^{\circ} \mathrm{C}$ in $95 \%$ humidity and $5 \% \mathrm{CO}_{2}$.

TSA treatment of Hep G2 cells. The HepG2 cells were seeded at $1 \times 10^{5}$ cells/well, in a 24 -well plate, and cultured in DMEM, supplemented with $10 \%$ FBS, overnight. The following day, the cells were treated with a final concentration of $670 \mathrm{nM}$ TSA (Sigma-Aldrich, St Louis, MO, USA) for 24 h (24). The TSA was dissolved in dimethyl sulfoxide (DMSO, Sigma-Aldrich) and diluted in fresh medium. The control well was seeded with the same cellular density and treated with DMSO diluted with phosphate-buffered saline. To determine the effects of TSA concentration and treatment duration on $e k 2 \alpha$ expression levels, the HepG2 cells were treated with the indicated doses of TSA for $24 \mathrm{~h}$, and at the indicated treatment durations with $750 \mathrm{nM}$ TSA. The cells were observed using an inverted microscope (Leica Microsystems GmbH, Wetzlar, Germany).

Total cellular RNA extraction and cDNA synthesis. Total RNA was extracted using the RNeasy Mini kit (Qiagen, Hilden, Germany). RNase-Free DNase I (Qiagen) was used to eliminate any contaminating genomic DNA. The yield and purity of the total RNA was assessed by measuring the absorbance at $260 \mathrm{~nm}$ using a BioPhotometer Plus (Eppendorf, Hamburg, Germany). The integrity and size distribution of the total RNA were examined by a $1 \%$ agarose gel electrophoresis. cDNA was reverse transcribed from $1 \mu \mathrm{g}$ of total RNA, using the RevertAid ${ }^{\mathrm{TM}} \mathrm{H}$ Minus First Strand cDNA Synthesis kit (Thermo Fisher Scientific, Waltham, MA, USA).

Preparation of plasmids as homologous standards for qPCR. Plasmids containing the coding sequences of the various $\mathrm{CK}$ and EK isoforms (pET14b-ck $\alpha$, pGEXRB-ck $\alpha 2, \mathrm{pET} 14 \mathrm{~b}-c k \beta$, pET14b-ek1, pMALK4-ek2 $\alpha$, and pGEXRB-ek2 $\beta$ ) were used as standards for the qPCR. The plasmid copy number (PCN) was calculated using the following equation, as described by previous methods (25):

$$
\begin{gathered}
\mathrm{PCN}=\left[\left(6.02 \times 10^{23} \mathrm{copy} / \mathrm{mol}\right) \times \mathrm{DNA} \text { amount }(\mathrm{g})\right] \\
/[\text { DNA length }(\mathrm{bp}) \times 660(\mathrm{~g} / \mathrm{mol} / \mathrm{bp})]
\end{gathered}
$$

A 10-fold serial dilution of each plasmid DNA, ranging from $1 \times 10^{2}$ to $1 \times 10^{7}$ copies $/ \mu 1$, was used to construct a standard curve. The PCR amplification efficiency of each gene was calculated from the corresponding standard curve using the following equation, as described previously (24):

$$
\mathrm{E}(\%)=\left(10^{-1 / \text { slope }}-1\right) \times 100 \%
$$

qPCR. A qPCR was performed using the ABI PRISM 7000 Sequence Detection system (Applied Biosystems Life Technologies, Foster City, CA, USA). Each reaction was performed in a $25 \mu \mathrm{l}$ volume containing $12.5 \mu \mathrm{l}$ Power SYBR $^{\circledR}$ Green I Master Mix (Applied Biosystems), specific primer (300 $\mathrm{nM}$ of target primer or $1 \mu \mathrm{l}$ of reference primer) and $1 \mu \mathrm{l}$ template DNA (1:2 diluted cDNA or plasmid DNA 
Table I. $c k$ and $e k$ primer sequences.

PCR

Primer name

Primer sequence

product size(bp)

$\begin{array}{ll}\text { total-cka } & \text { F 5'-TCAGAGCAAACATCCGGAAGT-3' } \\ & \text { R 5'-GGCGTAGTCCATGTACCCAAAT-3' } \\ c k \alpha 2 & \text { F 5'-GGCCTTAGCAACATGCTGTTC-3' } \\ & \text { R 5'-AGCTTGTTCAGAGCCCTCTTT-3' } \\ c k \beta & \text { F 5'-ATGTTCGCCATACTTGCGGA-3' } \\ & \text { R 5'-AATTGCGCCATCTTCGTGG-3' } \\ e k 1 & \text { F 5'-AAAGGTTCCTAAGTGATATCCC-3' } \\ & \text { R 5'-GCCAGGTAGTTGTATCCAGA-3' } \\ e k 2 \alpha & \text { F 5'-TTCAATGAGTTTGCAGGCGTG-3' } \\ & \text { R 5'-CAGAAGAAGTGAGACGCCAG-3' } \\ e k 2 \beta & \text { F 5'-TGTGTCTTCCACAATGACTGC-3' } \\ & \text { R 5'-TCGATGGTGGAGTACTGGTTC-3' }\end{array}$

F, forward; R, reverse; bp, base pairs; PCR, polymerase chain reaction.

dilutions ranging from $1 \times 10^{2}$ to $1 \times 10^{7}$ copies/ $\left.\mu 1\right)$. The specific primer sets for total-ck $\alpha, c k \alpha 2, c k \beta, e k 1, e k 2 \alpha$ and $e k 2 \beta$ (Table I) were designed to span intron-exon boundaries using in silico PCR and Primer-BLAST (National Institutes of Health, Bethesda, MA, USA). The reference genes used for relative quantification were UBC and YWHAZ. The reference gene primers were purchased from TATAA Biocenter (Göteburg, Sweden). The calculation used to determine the normalised relative quantities was previously described by Hellemans et al (26). Thermal cycling was performed using the default settings of the ABI PRISM 7000 SDS software 1.0 , as follows: $2 \mathrm{~min}$ at $50^{\circ} \mathrm{C}, 10 \mathrm{~min}$ at $95^{\circ} \mathrm{C}$, followed by 40 cycles of $10 \mathrm{sec}$ at $95^{\circ} \mathrm{C}$ and $1 \mathrm{~min}$ at $60^{\circ} \mathrm{C}$. A melt curve analysis was performed immediately following the amplification, with temperatures ranging between 60 and $95^{\circ} \mathrm{C}$ in $0.1^{\circ} \mathrm{C}$ increments, in order to verify the PCR specificity. Absolute quantification of YWHAZ was also performed for the control and TSA-treated cells in the experiments involving TSA. YWHAZ was included as a control to account for possible variations in overall gene expression levels as a result of TSA treatment.

Identification of $C p G$ islands in the gene promoter region. The location of $\mathrm{CpG}$ islands at the $c k \alpha, c k \beta, e k 1$ and $e k 2$ promoter regions were determined using $\mathrm{CpG}$ Plot, which is available from the European Molecular Biology Open Software Suite (http://www.ebi.ac.uk/emboss/cpgplot). A CpG island is defined as a region of $>200$ bp nucleotides, with a GC content $>50 \%$ and an observed/expected ratio $>0.6$.

Statistical analysis. Statistical evaluations were performed using a paired t-test and a one-way analysis of variance with the Tukey Honestly Significant Difference post-hoc test. All analyses were performed using SPSS version 18.0 (SPSS, Inc., Chicago, IL, USA). $\mathrm{P}<0.05$ was considered to indicate a statistically significant difference.

\section{Results}

Expression profiling of $c k$ and ek $m R N A$ variants in MCF7, HCT116 and HepG2 cells. In the present study, both absolute and relative quantification strategies were used to quantify and compare the mRNA expression levels of $c k$ and $e k$ variants in MCF7, HCT116 and HepG2 cells. The specificity of the PCR amplification of $c k$ and $e k$ variants was confirmed using a melt curve analysis and agarose gel electrophoresis. The slope of the generated standard curves for all of the amplicons was between -3.2 and -3.6 , confirming that the amplifications were highly efficient.

The comparative cycle threshold $\left(\mathrm{C}_{t}\right)$ method was used to determine the expression levels of total-ck $\alpha, c k \alpha 2$, $c k \beta, e k 1, e k 2 \alpha$ and $e k 2 \beta$ variants, relative to the MCF7 cells and normalised to the geometric means of UBC and YWHAZ (Fig. 1A). A previous study identified UBC and YWHAZ as the most suitable reference genes for normalisation in gene expression studies using MCF7, HCT116 and HepG2 cells (27). In addition, an absolute mRNA copy number of each $c k$ and $e k$ variant was calculated from respective standard curves (Fig. 1B). The $c k \alpha$ gene, which has previously been reported to be associated with carcinogenesis (7), had the highest mRNA expression levels detected in the HepG2 cells; total-ck $\alpha$ had a value of $2031 \pm 415.78$ mRNA copies/ng total RNA. The highest mRNA expression levels of $c k \beta$ and $e k 1$, were found in the HCT116 cells. The expression levels, relative to the MCF7 cells, were 2 - and 4-fold higher, respectively (Fig. 1A). The absolute copy numbers of the $c k \beta$ and $e k 1$ mRNA variants in the HCT116 cells, however, as determined by absolute quantification, were markedly lower. The $e k 2 \alpha$ variant displayed the highest mRNA expression levels in the MCF7 cells. $c k \alpha 2$ and $e k 2 \beta$, which are splice variants of $c k \alpha$ and $e k 2$ genes, respectively, were expressed at markedly low levels in the MCF7, HCT116 and HepG2 cells (Fig. 1B). The mRNA expression levels of $c k \alpha 2$ were as low as $2 \pm 1.41,2 \pm 0.71$ 
and $18 \pm 4.24 \mathrm{mRNA}$ copies/ng total RNA, as determined by absolute quantification; whereas $e k 2 \beta$ mRNA expression was $10 \pm 6.36,18 \pm 4.24$ and $1 \pm 0.71 \mathrm{mRNA}$ copies/ng total RNA in the MCF7, HCT116 and HepG2 cells, respectively.

In the present study, due to overlapping cDNA sequences, a general $c k \alpha$ primer pair, known as total-ck $\alpha$ and a specific $c k \alpha 2$ primer pair were designed to quantify the mRNA expression levels of the $c k \alpha$ variants in the MCF7, HCT116 and HepG2 cells. The $c k \alpha 1$ mRNA expression levels were quantified only by the absolute quantification strategy. The absolute mRNA copy numbers of the $c k \alpha l$ variants were determined by calculating the difference between the copy number of the total-ck $\alpha$ and $c k \alpha 2$ variants. Due to the markedly low expression levels of the $c k \alpha 2$ variant detected in all of the cancer cell lines tested, the mRNA copy number of the $c k \alpha l$ variant was shown as equal to the copy number of total-ck (Fig. 1B).

Besides determining the quantification of each $c k$ and $e k$ mRNA variant in various cancer cells, the qPCR approach permits the comparison of mRNA expression levels of all of the $c k$ and $e k$ variants in the same cancer cell line. For the relative quantification strategy, the $c k \beta$ gene was chosen as the calibrator and its relative mRNA expression was arbitrarily set at 1.0. The mRNA expression levels of total-ck $\alpha$, $c k \alpha 2, e k 1, e k 2 \alpha$ and $e k 2 \beta$ were therefore compared, relative to $c k \beta$ (Fig. 2A). The $e k 2 \alpha$ variant had the highest mRNA expression levels among $c k$ and $e k$ variants, in MCF7 cells, as determined by absolute quantification, with a difference of $2762 \mathrm{mRNA}$ copies/ng total RNA, as compared with $c k \beta$ (Fig. 2B). This finding is concordant with the 2.9 -fold higher expression level of $e k 2 \alpha$, as compared with $c k \beta$, as determined by the relative quantification strategy (Fig. 2A). The mRNA expression levels of $c k \alpha l$ were slightly lower, as compared with $c k \beta$, in the MCF7 cells with 248 copies difference between total $c k \alpha 1$ and $c k \beta$ mRNA expression levels, as determined by the absolute quantification strategy. In the HCT116 cells, the mRNA expression levels of $c k \beta$ were the highest, with $1975 \pm 695.93$ mRNA copies/ng total RNA. The relative mRNA expression levels of total-ck $\alpha$ were 0.59 -fold lower, as compared with $c k \beta$. Conversely, the expression levels of total-ck $\alpha$ were the highest, as compared with the other $c k$ and $e k$ variants, in the HepG2 cells. The expression of total- $c k \alpha$ was 1.26-fold higher, as compared with $c k \beta$; whereas the relative $e k 1$ mRNA expression level was $\sim 50 \%$ that of $c k \beta$. The $e k 2 \alpha$ mRNA expression level was 0.08 -fold, as compared with $c k \beta$, which was equal to $111 \pm 39.85 \mathrm{mRNA}$ copies/ng total RNA, in the HepG2 cells. The mRNA expression levels of the $c k \alpha 2$ and $e k 2 \beta$ variants in the MCF7, HCT116 and HepG2 cells were negligible as compared with $c k \beta$.

Prediction of $C p G$ islands at $c k$ and ek gene promoters. The presence of $\mathrm{CpG}$ islands at the gene promoter region potentially increases the DNA methylation activities, which may affect the expression of $c k$ and $e k$ genes. To examine this possibility, the $c k \alpha, c k \beta, e k 1$ and $e k 2$ promoter regions were analysed for the presence of $\mathrm{CpG}$ islands. The promoter regions of $c k \alpha, c k \beta$ and $e k 2$ were all predicted to contain putative $\mathrm{CpG}$ islands (Fig. 3). This hypothesis was supported by the relatively high GC content $(>70 \%)$ and an observed/expected ratio of $>0.70$. An analysis of the $c k \alpha$ gene promoter region predicted two $\mathrm{CpG}$ islands
$\mathbf{A}$

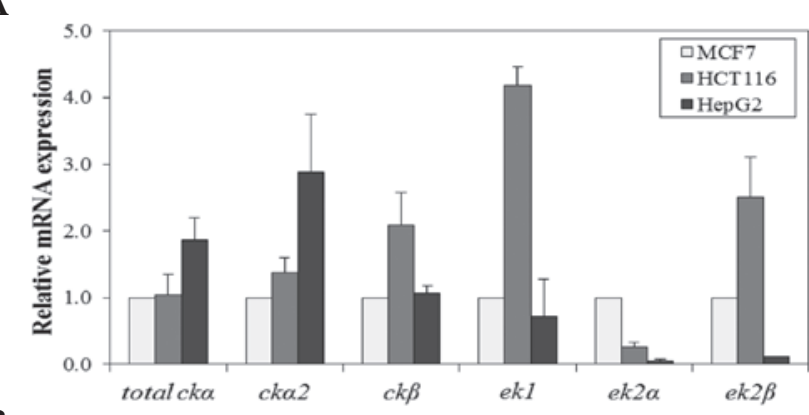

B

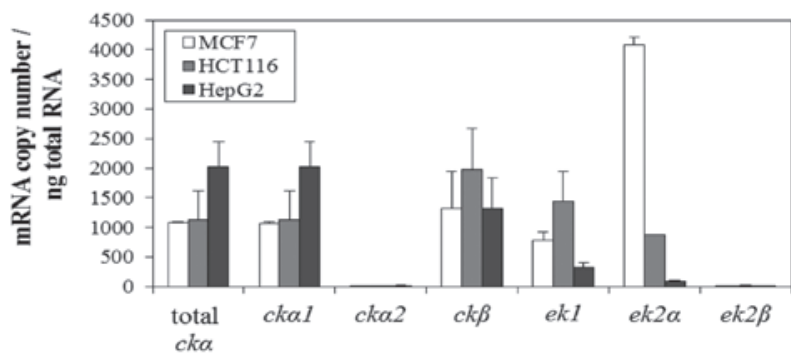

Figure 1. Expression profiling of $c k$ and $e k$ mRNA variants in MCF7 breast, HCT116 colon and HepG2 liver cancer cells. (A) Relative quantification of mRNA expression levels of $c k$ and $e k$ variants in the three cancer cell lines, relative to MCF7 cells, post-normalisation to the geometric means of UBC and YWHAZ reference genes. (B) Absolute quantification of mRNA expression levels of $c k$ and $e k$ variants in copy number/ng total RNA in MCF7, HCT116 and HepG2 cells. Each bar represents the means \pm standard deviation of three independent experiments.

A
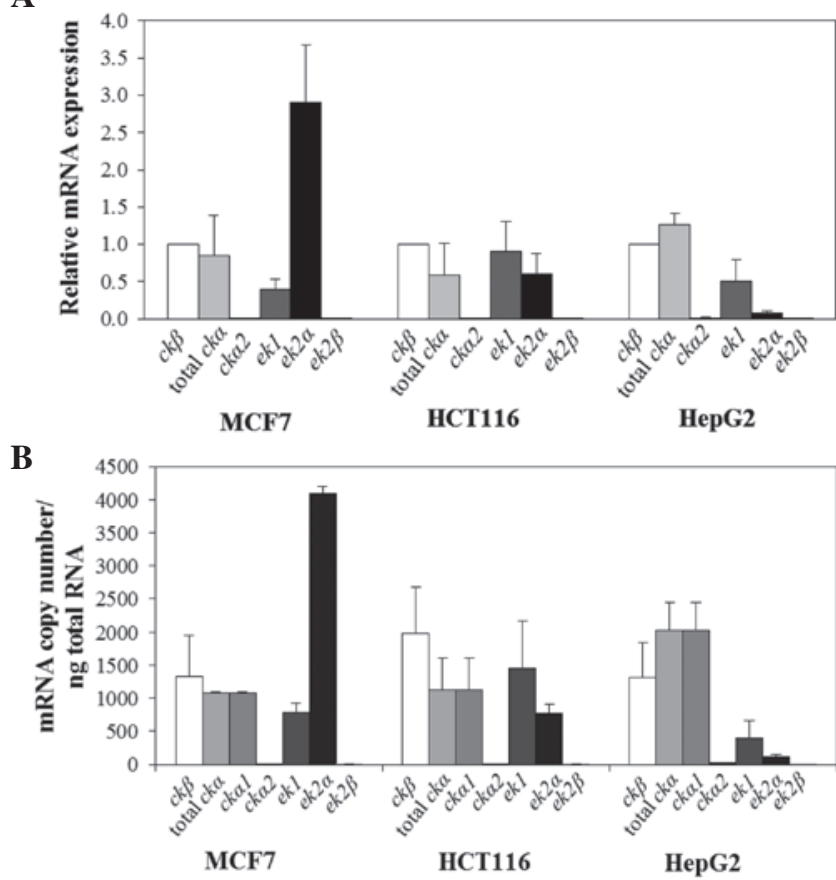

Figure 2. Comparison of $c k$ and $e k$ mRNA expression levels in MCF7 breast, HCT116 colon and HepG2 liver cancer cells. (A) Relative and (B) absolute quantifications of total-ck $\alpha, c k \alpha 2, e k 1, e k 2 \alpha$ and $e k 2 \beta$ mRNA variants in the three cell lines. Each bar represents the means \pm standard deviation of three independent experiments.

located at the 5' upstream regions between -695 and -907 (213 bp) and between -55 and -566 (512 bp). The former $\mathrm{CpG}$ island contained $138 \mathrm{CpGs}$, a GC content of $64.79 \%$ and 


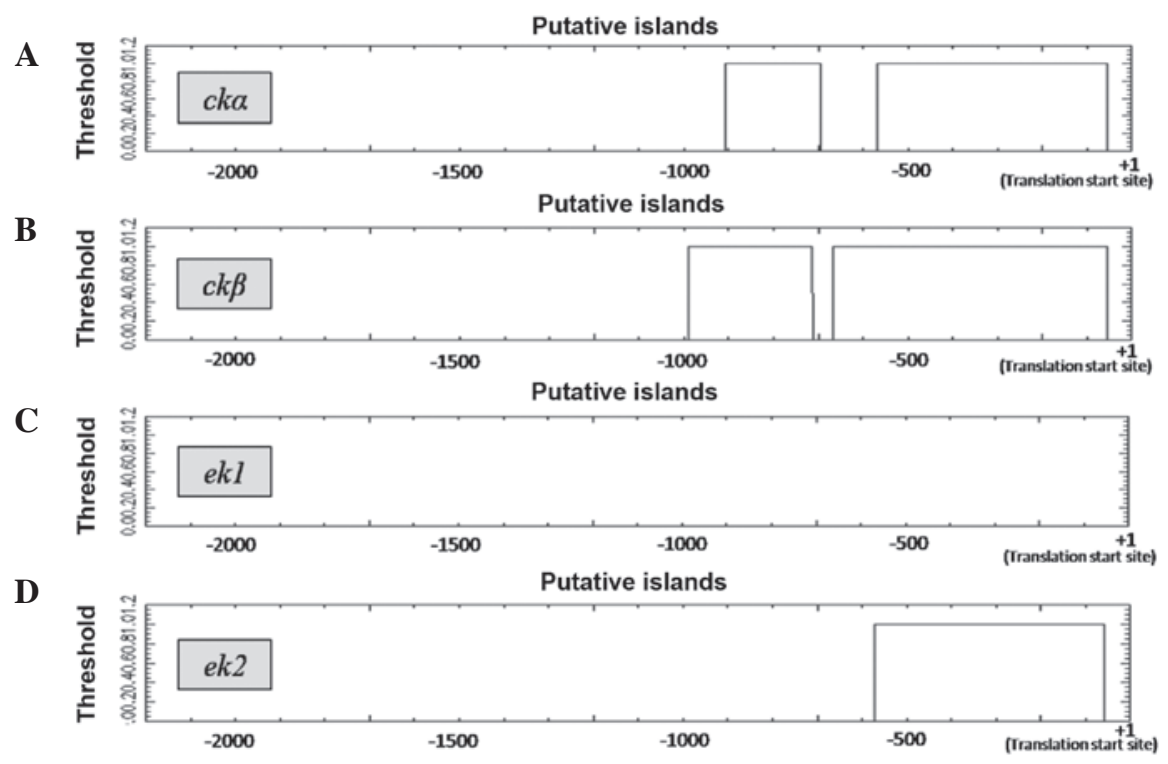

Figure 3. Prediction of $\mathrm{CpG}$ islands at the promoter regions of the $c k \alpha, c k \beta, e k 1$ and $e k 2$ genes, as determined by CpG Plot.

an observed/expected ratio of 0.76 , whereas the latter $\mathrm{CpG}$ island consisted of $405 \mathrm{CpGs}$, a GC content of $79.10 \%$ and an observed/expected ratio of 0.96 . Similarly, two $\mathrm{CpG}$ islands were predicted at the gene promoter region of $c k \beta$. The first $\mathrm{CpG}$ island region was located between -712 and -988 (277 bp), consisted of $164 \mathrm{CpGs}$, had a $59.21 \%$ GC content and an observed/expected ratio of 0.73 . The other $\mathrm{CpG}$ island covered a larger region, between -55 and -665 (611 bp), consisted of $437 \mathrm{CpGs}$ with a $71.52 \% \mathrm{GC}$ content and an observed/expected ratio of 0.94 . A $512 \mathrm{bp} \mathrm{CpG}$ island between -59 and -570 containing $398 \mathrm{CpGs}$, with a $77.73 \% \mathrm{GC}$ content and an observed/expected ratio of 0.80 was predicted in the $e k 2$ promoter region. There were no predicted $\mathrm{CpG}$ islands at the $5^{\prime}$ upstream region of the $e k l$ gene. These results indicate that the regulation of $c k$ and $e k$ expression may be affected by DNA methylation at the promoter region. This prompted the exploration into the effects of DNA demethylation on the expression levels of $c k$ and $e k$ mRNA variants.

Effects of TSA treatment on ck and ek mRNA expression profiles. The HDAi TSA was used to investigate the effects of DNA methylation on $c k$ and $e k$ mRNA expression. The expression levels were determined using the absolute quantification strategy, with YWHAZ reference gene used as the internal control. TSA, which indirectly causes DNA demethylation, altered total- $c k \alpha, c k \beta$ and $e k 2 \alpha$ mRNA expression levels in the HepG2 cells (Fig.4). The mRNA expression levels of total-ck $\alpha$, which were high in the HepG2 cells, exhibited a significant upregulation in response to TSA treatment, as compared with the control $(\mathrm{P}<0.05)$. Conversely, TSA treatment resulted in a substantial downregulation in the mRNA expression levels of $c k \beta$, with a $>2$-fold decrease to $438 \pm 198.01 \mathrm{mRNA}$ copies/ng total RNA, as compared with the controlexpressionof $1059 \pm 32.95 \mathrm{mRNAcopies} /$ ngtotalRNA $(\mathrm{P}<0.05)$. There were no significant changes to the $e k l$ mRNA expression levels in the HepG2 cells, in response to the TSA treatment. This observation correlates with the absence of a predicted $\mathrm{CpG}$ island in the $e k l$ promoter region. Notably,

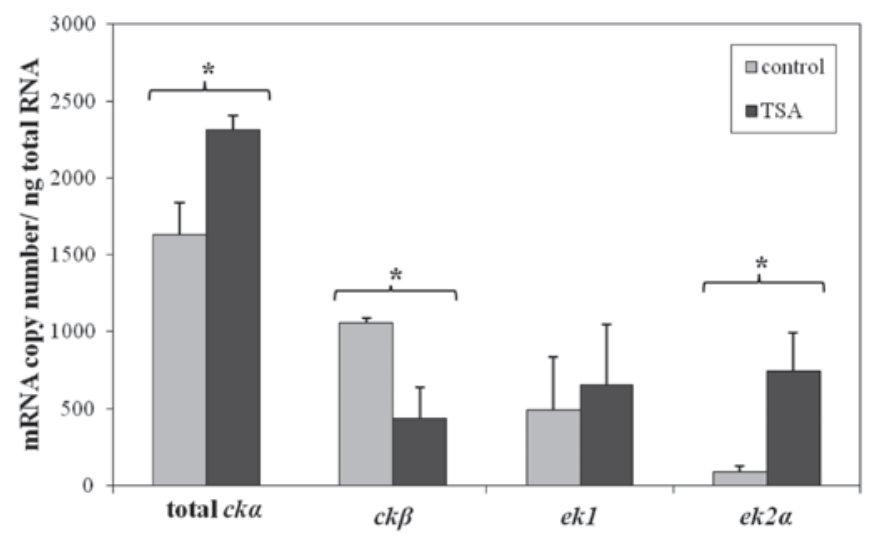

Figure 4. Effects of trichostatin A (TSA) treatment on the mRNA expression levels of total-ck $\alpha, c k \beta, e k 1$ and $e k 2 \alpha$ in the HepG2 liver cancer cells. Each bar represents the means \pm standard deviation of three independent experiments. A paired $t$-test was used to compare the mRNA expression levels of the control and TSA treated groups. ${ }^{*} \mathrm{P}<0.05$.

the mRNA expression levels of $e k 2 \alpha$, which were repressed in the HepG2 cells, exhibited a substantial increase of $>8$ fold to 744 mRNA copies/ng total RNA, in response to TSA treatment.

Effects of TSA treatment dosage and duration on ek2 $\alpha$ $m R N A$ expression. The substantial induction of $e k 2 \alpha \mathrm{mRNA}$ expression in response to TSA treatment, led to further investigations regarding the effects of various TSA concentrations and treatment durations on $e k 2 \alpha$ expression levels. A TSA concentration as low as $250 \mathrm{nM}$ was able to significantly induce $e k 2 \alpha$ mRNA expression in the HepG2 cells (Fig. 5). A dose-dependent increase in the mRNA expression levels of $e k 2 \alpha$ was observed in response to TSA treatment up to $1000 \mathrm{nM}$. At $1000 \mathrm{nM}$ TSA, the expression of $e k 2 \alpha$ was $666 \pm 46.73 \mathrm{mRNA}$ copies/ng total RNA, as compared with the control of $101 \pm 11.27$ mRNA copies/ng total RNA. There were no significant differences in the mRNA expression 


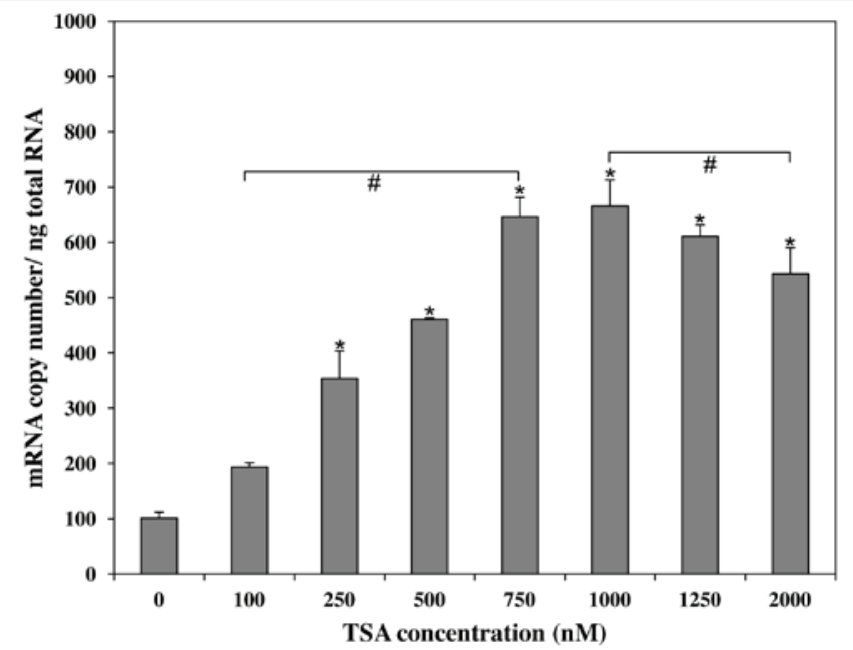

Figure 5. Effects of trichostatin A (TSA) concentration on ek $2 \alpha$ mRNA expression levels in the HepG2 liver cancer cells. The cells were exposed to the indicated concentrations of TSA for $24 \mathrm{~h}$. Each bar represents the mean \pm standard deviation of three independent experiments. Statistical analysis was performed using one-way analysis of variance with Tukey's HSD post-hoc test $\left({ }^{*} \mathrm{P}<0.05\right.$ vs. control at $0 \mathrm{mM}$; ${ }^{\#} \mathrm{P}<0.05$, significant between different concentrations)

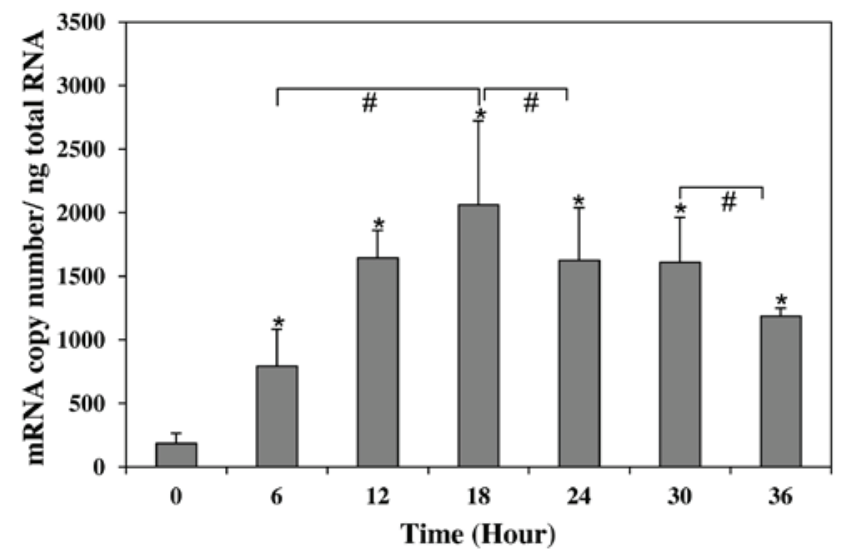

Figure 6. Effects of trichostatin A (TSA) exposure duration on ek $2 \alpha$ mRNA expression levels in HepG2 liver cancer cells. The cells were exposed to $750 \mathrm{nM}$ TSA for $6,12,18,24,30$ and $36 \mathrm{~h}$. Each bar represents the mean \pm standard deviation of three independent experiments. Statistica analysis was performed using one-way analysis of variance with Tukey's HSD post-hoc test ( ${ }^{*} \mathrm{P}<0.05$ vs. control at $0 \mathrm{~h} ;{ }^{~} \mathrm{P}<0.05$, significant between different treatment durations).

levels of $e k 2 \alpha$ between 750 and $1000 \mathrm{nM}$ TSA (P>0.05). Therefore, a TSA concentration of $750 \mathrm{nM}$ was used for the subsequent experiment.

TSA treatment also induced $e k 2 \alpha$ mRNA expression, in the HepG2 cells, in a time-dependent manner (Fig. 6). A significant increase in the mRNA expression levels of $e k 2 \alpha$, in response to TSA treatment, was observed throughout the duration tested, starting from $6 \mathrm{~h}(\mathrm{P}<0.05)$. The highest $e k 2 \alpha$ mRNA expression levels were detected $18 \mathrm{~h}$ post-TSA treatment with 2061 mRNA copies/ng total RNA, as compared with 184 mRNA copies/ng total RNA in the control. The longer treatment durations, $>18 \mathrm{~h}$, resulted in a gradual decrease in the mRNA expression levels of $e k 2 \alpha$.

\section{Discussion}

Two qPCR quantification approaches, absolute and relative, are usually applied to determine the mRNA expression levels of $c k$ and $e k$ variants. Different experimental protocols are required for these approaches and each of them has advantages over the other. Relative quantification takes into account the expression levels of target genes, as well as the reference gene, therefore excluding the need to use a calibration curve. However, the use of a stably expressed reference gene throughout the experiment is required and must be identified prior to quantification. To perform a reliable comparison, the amplification efficiencies of both the target and reference genes must be similar (28). Normalisation with a suitable reference gene greatly minimises the variation in total RNA input, qPCR efficiencies and sample to sample variation; therefore, producing accurate and reliable results.

Conversely, normalization to a reference gene is not required in the absolute quantification strategy. The absolute quantification approach uses a highly reproducible calibration curve, which is generated using a serial dilution of a standard DNA molecule, such as recombinant plasmid DNA, which was used in the present study. The absolute quantification strategy is dependent on the accuracy of the standard; therefore, similar qPCR efficiencies for both the calibration curve and target gene, as well as the accurate determination of the concentration of the standard, are of critical importance. Absolute quantification allows the absolute copy number of the mRNA transcript to be determined, in contrast to relative quantification which only measures the relative change to the mRNA expression level. A wide dynamic range of the constructed calibration curve, up to a few orders of magnitude, also allows genes that are very lowly expressed to be quantified.

In the present study, quantification of the mRNA expression levels of the $c k$ and $e k$ variants in MCF7, HCT116 and HepG 2 cells was determined using both the absolute and relative qPCR approaches, which generated similar results. In relative quantification, application of a mathematical model with a correction for the PCR kinetic efficiency (26) has previously been shown to reflect the actual cell situation, therefore allowing the generation of reliable results similar to absolute quantification (29). The relative quantification strategy provides a ratio of the expression of the gene of interest, rather than the absolute copy number in a defined concentration of total RNA. In the present study, each ratio was generated based on the expression of total-ck $\alpha, c k \alpha 2$, $c k \beta, e k 1, e k 2 \alpha$ and $e k 2 \beta$, normalised to the geometric means of UBC and YWHAZ expression levels. However, a high ratio produced in relative quantification does not necessarily reflect a high expression level of the target gene. Pfaffl (30) stated that these expression ratios are sensitive and dependent on the expression levels of the genes used for normalization. Therefore, the outcomes derived from relative and absolute qPCR strategies are not completely comparable. This may explain the differences observed in the mRNA expression levels of $c k \beta, e k 1, e k 2 \alpha$ and $e k 2 \beta$ determined using both approaches. For example, the mRNA expression levels of $e k 2 \beta$ in the HCT116 cells were shown to be $>2$-fold higher, as compared with the expression levels detected in the MCF7 
cells, as determined by the relative quantification strategy. However, the mRNA expression levels of $e k 2 \beta$ were only different by a small number of copies in the HCT116 and MCF7 cells, when the absolute quantification approach was used.

The results obtained from both quantification strategies supported the earlier hypothesis that the mRNA expression levels of each $c k$ and $e k$ variants in MCF7, HCT116 and HepG 2 cells varies. Total-ck $\alpha$ mRA expression was detected in all three cancer cell lines tested. Notably, $c k \alpha$, which has previously been shown to be overexpressed in breast cancer-derived cell lines $(12,14)$, was expressed at lower expression levels in the MCF7 breast cancer cells, as compared with the HepG2 liver cancer cells in the present study. Currently, there have been few comparisons made between $c k \alpha$ and $\operatorname{ck} \beta$ mRNA expression levels in cancer cells. The present study demonstrated that $c k \beta$ transcript expression levels exceed that of total $c k \alpha$ in breast and colon cancer cell lines. This observation is in agreement with a previous qPCR analysis of $c k \alpha$ and $c k \beta$ mRNA expression ratios in T47D human breast cancer cells, that showed higher mRNA expression levels of $c k \beta$ (10). The balance of $c k \alpha$ and $c k \beta$ mRNA expression levels has also been shown to have a vital role in cancer cell survival (15).

EK1 protein was previously demonstrated as being uniformly distributed in various human tissues, whereas EK2 was selectively expressed in the kidney, liver, ovary, testis and prostate (6). Tian et al (31) detected significantly higher $e k 2$ mRNA expression levels in the mouse liver, as compared with the kidney and testis. In the present study, $e k 2 \beta$, the splice variant of $e k 2$, exhibited a markedly low mRNA transcript level in all three of the cancer cell lines examined, thus suggesting that transcription of the $e k 2 \beta$ gene may be negligible and this isoform may not have a significant role in phosphatidyletanolamine synthesis in these three cancer cell lines.

The present study demonstrated that TSA treatment did not have the same effects on the gene expression of all of the $c k$ and $e k$ variants. This is concordant with previous studies, which showed that inhibition of HDAC activity by HDACi affected only $2-5 \%$ of expressed genes in numerous cancer cell lines (32-34). The presence of $\mathrm{CpG}$ islands was predicted at the promoter regions of $c k \alpha, c k \beta$ and $e k 2 \alpha$, which may explain the effects of TSA treatment on the expression of these genes. It has previously been stated that $94 \%$ of TSA-induced genes have $\mathrm{CpG}$ islands at the gene promoter region (35). It may be hypothesized that TSA influences the gene expressions of $c k$ and $e k$ by DNA demethylation at the methylated $\mathrm{CpG}$ islands in the promoter region, as is the case for p16, SALL3 and GATA4 genes (36).

Treatment with various HDACi, such as vorinostat, in a human myeloma cell line was shown to increase the degree of acetylation and methylation of lysines in histones $\mathrm{H} 3$ and $\mathrm{H} 4$ at the proximal promoter region of the target gene (37). Chromatin immunoprecipitation analysis previously detected an accumulation of acetylated histones $\mathrm{H} 3$ and $\mathrm{H} 4$ at the promoter regions of the upregulated genes post-TSA treatment (38). Therefore, it may be possible that the elevated $e k 2 \alpha$ mRNA expression was due to the accumulation of acetylated histones $\mathrm{H} 3$ and $\mathrm{H} 4$, located around the promoter region, which subsequently induced the expression of $e k 2 \alpha$. Furthermore, TSA may also induce the recruitment of euchromatic markers and RNA polymerase II to the transcription factor complex that binds to the promoter, thus facilitating gene transcription (39).

Previously, magnetic resonance spectroscopy demonstrated that the HDACis LAQ824 and SAHA, increased the cellular levels of PCho in human colon cancer cells and tumor xenografts (40). The rise in PCho de novo synthesis was shown to be closely associated with an induction of $c k \alpha$ expression. The present study showed that the mRNA expression levels of $c k \alpha$ in HepG2 cells were upregulated in response to TSA treatment, and supported the use of PCho as a potential biomarker to monitor the activities of HDACi.

The downregulation of $c k \beta$ expression upon TSA treatment, as observed in the present study, may be the result of either a direct or indirect inhibitory effect. The indirect effect of TSA on HDAC regulated gene transcription may be through specific recruitment of non-histone HDAC targets to various gene regulatory regions (33). The attenuating effects on $c k \beta$ expression may result in a reduction in cellular $\mathrm{CK}$ activity and phosphatidylcholine biosynthesis in liver cancer cells. However, the enhanced expression of total $c k \alpha$ by TSA would compensate for phosphatidylcholine biosynthesis which is required for cell proliferation, especially during tumor progression.

The dose- and time-dependent induction of $e k 2 \alpha$ by TSA is similar to previous reports that showed the variations in TSA dose and treatment duration that altered the expression profile of genes involved in apoptosis and the cell cycle $(38,41)$. The mRNA expression levels of genes affected by HDAC inhibition have been shown to increase proportionally with the concentration and treatment duration of HDACi (42).

The mRNA expression levels of $c k$ and $e k$ variants in MCF7, HCT116 and HepG2 cells were determined and quantified in the present study. Both relative and absolute quantification strategies generated similar mRNA expression patterns. In response to epigenetic drug treatment, TSA upregulated the expression levels of total-ck $\alpha$ and $e k 2 \alpha$, but downregulated the expression levels of $c k \beta$. The induction of $e k 2 \alpha$ by TSA was both concentration- and time-dependent. The effects of TSA may be mediated by the presence of $\mathrm{CpG}$ islands in the promoter regions of the affected genes. However, further experiments are required to confirm this assumption. A future aim may be to identify the $\mathrm{CpG}$ sites that are responsible for the pronounced effects on $c k \alpha, c k \beta$ and $e k 2 \alpha$ gene expressions in HepG 2 cells during TSA treatment.

\section{Acknowledgements}

The present study was funded by the Research University Grant (no. 1001/PPSK/813034, 1001/PPSK/815101) from the Universiti Sains Malaysia. Chua Siang Ling was supported by a fellowship from the Institute of Postgraduate Studies, Universiti Sains Malaysia. The authors of the present study would also like to acknowledge the technical support provided by the UPM, Cell Culture and Biomedicine Laboratories, School of Health Sciences, Universiti Sains Malaysia. 


\section{References}

1. Kennedy EP: Metabolism of lipides. Annu Rev Biochem 26: 119-148, 1957.

2. Aoyama C, Liao H and Ishidate K: Structure and function of choline kinase isoforms in mammalian cells. Prog Lipid Res 43: 266-281, 2004.

3. Aoyama C, Yamazaki N, Terada H and Ishidate K: Structure and characterization of the genes for murine choline/ethanolamine kinase isozymes alpha and beta. J Lipid Res 41: 452-464, 2000.

4. Yamazaki N, Yamanaka Y, Hashimoto Y, Shinohara Y, Shima A and Terada H: Structural features of the gene encoding human muscle type carnitine palmitoyltransferase I. FEBS Lett 409: 401-406, 1997.

5. Yamazaki N, Shinohara Y, Kajimoto K, Shindo $M$ and Terada H: Novel expression of equivocal messages containing both regions of choline/ethanolamine kinase and muscle type carnitine palmitoyltransferase I. J Biol Chem 275: 31739-31746, 2000.

6. Lykidis A, Wang J, Karim MA and Jackowski S: Overexpression of a mammalian ethanolamine-specific kinase accelerates the CDP-ethanolamine pathway. J Biol Chem 276: 2174-2179, 2001.

7. Ramírez de Molina A, Sarmentero-Estrada J, Belda-Iniesta C, Tarón M, Ramírez de Molina V, Cejas P, Skrzypski M, GallegoOrtega D, de Castro J, Casado E, et al: Expression of choline kinase alpha to predict outcome in patients with early-stage non-small-cell lung cancer: a retrospective study. Lancet Oncol 8: 889-897, 2007.

8. Al-Saffar NM, Troy H, Ramírez de Molina A, et al: Noninvasive magnetic resonance spectroscopic pharmacodynamic markers of the choline kinase inhibitor MN58b in human carcinoma models. Cancer Res 66: 427-434, 2006.

9. Glunde K and Serkova NJ: Therapeutic targets and biomarkers identified in cancer choline phospholipid metabolism. Pharmacogenomics 7: 1109-1123, 2006.

10. Eliyahu G, Kreizman T and Degani H: Phosphocholine as a biomarker of breast cancer: molecular and biochemical studies. Int J Cancer 120: 1721-1730, 2007.

11. Katz-Brull R, Lavin PT and Lenkinski RE: Clinical utility of proton magnetic resonance spectroscopy in characterizing breast lesions. J Natl Cancer Inst 94: 1197-1203, 2002.

12. Ramírez de Molina A, Sarmentero-Estrada J, Belda-Iniesta C, et al: Expression of choline kinase alpha to predict outcome in patients with early-stage non-small-cell lung cancer: a retrospective study. Lancet Oncol 8: 889-897, 2007.

13. Too WC, Wong MT, Few LL and Konrad M: Highly specific antibodies for co-detection of human choline kinase $\alpha 1$ and $\alpha 2$ isoforms. PLoS One 5: e12999, 2010.

14. Gallego-Ortega D, Ramirez de Molina A, Ramos MA, et al: Differential role of human choline kinase alpha and beta enzymes in lipid metabolism: implications in cancer onset and treatment. PLoS One 4: e7819, 2009.

15. Gruber J, See Too WC, Wong MT, Lavie A, McSorley T and Konrad M: Balance of human choline kinase isoforms is critical for cell cycle regulation: implications for the development of choline kinase-targeted cancer therapy. FEBS J 279: 1915-1928, 2012.

16. Kiss Z: Regulation of mitogenesis by water-soluble phospholipid intermediates. Cell Signal 11: 149-157, 1999.

17. Juan LJ, Shia WJ, Chen MH, et al: Histone deacetylases specifically down-regulate p53-dependent gene activation. J Biol Chem 275: 20436-20443, 2000.

18. Meng J, Zhang HH, Zhou CX, Li C, Zhang F and Mei QB: The histone deacetylase inhibitor trichostatin A induces cell cycle arrest and apoptosis in colorectal cancer cells via p53-dependent and -independent pathways. Oncol Rep 28: 384-388, 2012.

19. Gang H, Dhingra R, Wang Y, Mughal W, Gordon JW and Kirshenbaum LA: Epigenetic regulation of E2F-1-dependent Bnip3 transcription and cell death by nuclear factor- $\kappa \mathrm{B}$ and histone deacetylase-1. Pediatr Cardiol 32: 263-266, 2011.

20. Furumai R, Ito A, Ogawa K, et al: Histone deacetylase inhibitors block nuclear factor- $\kappa \mathrm{B}$-dependent transcription by interfering with RNA polymerase II recruitment. Cancer Sci 102: 1081-1087, 2011.

21. Schnur N, Seuter S, Katryniok C, Rådmark O and Steinhilber D: The histone deacetylase inhibitor trichostatin A mediates upregulation of 5-lipoxygenase promoter activity by recruitment of Sp1 to distinct GC-boxes. Biochim Biophys Acta 1771: 1271-1282, 2007.
22. Xenidis N, Markos V, Apostolaki S, et al: Clinical relevance of circulating CK-19 mRNA-positive cells detected during the adjuvant tamoxifen treatment in patients with early breast cancer. Ann Oncol 18: 1623-1631, 2007.

23. Selker EU: Trichostatin A causes selective loss of DNA methylation in Neurospora. Proc Natl Acad Sci USA 95: 9430-9435, 1998.

24. Chiba T, Yokosuka O, Arai M, et al: Identification of genes up-regulated by histone deacetylase inhibition with cDNA microarray and exploration of epigenetic alterations on hepatoma cells. J Hepatol 41: 436-445, 2004.

25. Fu J, Li D, Xia S, et al: Absolute quantification of plasmid DNA by real-time PCR with genomic DNA as external standard and its application to a biodistribution study of an HIV DNA vaccine. Anal Sci 25: 675-680, 2009.

26. Hellemans J, Mortier G, De Paepe A, Speleman F and Vandesompele J: qBase relative quantification framework and software for management and automated analysis of real-time quantitative PCR data. Genome Biol 8: R19, 2007.

27. Chua SL, See Too WC, Khoo BY and Few LL: UBC and YWHAZ as suitable reference genes for accurate normalisation of gene expression using MCF7, HCT116 and HepG2 cell lines. Cytotechnology 63: 645-654, 2011.

28. Livak KJ and Schmittgen TD: Analysis of relative gene expression data using real-time quantitative PCR and the 2(-Delta Delta C(T)) Method. Methods 25: 402-408, 2001.

29. Chini V, Foka A, Dimitracopoulos G and Spiliopoulou I: Absolute and relative real-time PCR in the quantification of tst gene expression among methicillin-resistant Staphylococcus aureus: evaluation by two mathematical models. Lett Appl Microbiol 45: 479-484, 2007.

30. Pfaffl MW: Quantification strategies in real-time PCR. In: A-Z of Quantitative PCR. Bustin SA (ed). IUL Biotechnology Series, International University Line, La Jolla, pp89-120, 2004.

31. Tian Y, Jackson P, Gunter C, Wang J, Rock CO and Jackowski S: Placental thrombosis and spontaneous fetal death in mice deficient in ethanolamine kinase 2. J Biol Chem 281: 28438-28449, 2006.

32. Mitsiades CS, Mitsiades NS, McMullan CJ, et al: Transcriptional signature of histone deacetylase inhibition in multiple myeloma: biological and clinical implications. Proc Natl Acad Sci USA 101: 540-545, 2004.

33. Reid G, Métivier R, Lin CY, et al: Multiple mechanisms induce transcriptional silencing of a subset of genes, including oestrogen receptor alpha, in response to deacetylase inhibition by valproic acid and trichostatin A. Oncogene 24: 4894-4907, 2005.

34. Richon VM, Sandhoff TW, Rifkind RA and Marks PA: Histone deacetylase inhibitor selectively induces p21WAF1 expression and gene-associated histone acetylation. Proc Natl Acad Sci USA 97: 10014-10019, 2000.

35. Heller G, Schmidt WM, Ziegler B, et al: Genome-wide transcriptional response to 5-aza-2'-deoxycytidine and trichostatin a in multiple myeloma cells. Cancer Res 68: 44-54, 2008.

36. Wu LP, Wang X, Li L, et al: Histone deacetylase inhibitor depsipeptide activates silenced genes through decreasing both $\mathrm{CpG}$ and $\mathrm{H} 3 \mathrm{~K} 9$ methylation on the promoter. Mol Cell Biol 28: 3219-3235, 2008.

37. Gui CY, Ngo L, Xu WS, Richon VM and Marks PA: Histone deacetylase (HDAC) inhibitor activation of p21WAF1 involves changes in promoter-associated proteins, including HDAC1. Proc Natl Acad Sci USA 101: 1241-1246, 2004.

38. Chiba T, Yokosuka O, Fukai K, et al: Cell growth inhibition and gene expression induced by the histone deacetylase inhibitor, trichostatin A, on human hepatoma cells. Oncology 66: 481-491, 2004.

39. Nunes MJ, Milagre I, Schnekenburger M, Gama MJ, Diederich M and Rodrigues E: Sp proteins play a critical role in histone deacetylase inhibitor-mediated derepression of CYP46A1 gene transcription. J Neurochem 113: 418-431, 2010.

40. Beloueche-Babari M, Arunan V, Troy $\mathrm{H}$, et al Histone deacetylase inhibition increases levels of choline kinase $\alpha$ and phosphocholine facilitating noninvasive imaging in human cancers. Cancer Res 72: 990-1000, 2012.

41. Yang X, Phillips DL, Ferguson AT, Nelson WG, Herman JG and Davidson NE: Synergistic activation of functional estrogen receptor (ER)-alpha by DNA methyltransferase and histone deacetylase inhibition in human ER-alpha-negative breast cancer cells. Cancer Res 61: 7025-7029, 2001.

42. Marks PA: Histone deacetylase inhibitors: a chemical genetics approach to understanding cellular functions. Biochim Biophys Acta 1799: 717-725, 2010. 